We agree that there is no evidence to support the use of dexamethasone in meningococcal septicaemia. As previously highlighted, we did not find any evidence of benefit when it was used in meningococcal meningitis either. We reiterate that further clinical studies are urgently required to clarify these important issues.

CS NANAYAKKARA Consultant paediatrician

$\mathrm{R} \operatorname{COX}$

Paediatric Directorate,

Consultant microbiologist

Kettering General Hospital NHS Trust,

Kettering,

Northamptonshire NN16 8UZ

1 Calman KC. Meningococcal infection: meningitis and septicaemia London: Department of Health, 1994. (PL CMO (94)2.) 2 Control of meningococcal disease. Communicable Disease Report 1993;3:229-30.

\section{Risk factors for breast cancer}

\section{Socioeconomic differences might be explained by body mass}

EDITOR,-Andrew G Carnon and colleagues could not explain socioeconomic differences in survival from breast cancer by difference in tumour stage or biology. ${ }^{1}$ An alternative explanation might lie in differences in body mass. Heavier women have a worse prognosis from breast cancer than lighter women. ${ }^{2}$ Also, heavier women are less likely to attend screening programmes than lighter women. ${ }^{3}$ Therefore heavy women may be in double jeopardy of dying of breast cancer: they are less likely to attend for screening, so cancer is diagnosed later and when it is diagnosed the prognosis is poor. Given that social status is inversely correlated with body weight, ${ }^{4}$ differences in survival among social groups after breast cancer has been diagnosed may be attributable simply to class differences in body mass. Furthermore, the phenomenon whereby women who do not attend breast screening programmes have a higher mortality from the disease than those who do attend, ${ }^{5}$ even though the incidence of disease is similar, may again be explained by the increased body mass of those who do not attend relative to those who do.

\section{DAVID TORGERSON} Research fellow

Health Economics and Health Services Research Units,

University of Aberdeen,

Aberdeen AB9 2ZD

1 Carnon AG, Ssemwogerere A, Lamont DW, Hole DJ, Mallon $\mathrm{EA}$, George WD, et al. Relation between socioeconomic deprivation and pathological prognostic factors in women with breast cancer. $B M Y$ 1994;309:1054-7. (22 October.)

2 Bastarrachea J, Hortobagyi GN, Smith TL, Shu-Wan C, Buzdar $\mathrm{AU}$. Obesity as an adverse prognostic factor for patients receiving adjuvant chemotherapy for breast cancer. Ann Inter Med 1994;119:18-25.

3 Torgerson DJ, Donaldson C, Garton MJ, Russell IT, Westland M, Reid DM. Population screening for osteoporosis: do nonattenders have a lower risk of osteoporosis? Osteoporosis International 1994;4:149-53.

4 West R. Obesity. London: Office of Health Economics, 1994.

5 UK Trial of Early Detection of Breast Cancer Group. First results on mortality reduction in the UK trial of early detection of on mortality reduction in the UK
breast cancer. Lancet 1988;ii:411-6.

\section{Pollutants and pesticides may be important}

EDrToR,-K McPherson and colleagues fail to mention xeno-oestrogens and progesterone deficiency as important risk factors for breast cancer. ${ }^{1}$ Recently, several pollutants and pesticides have been recognised as having potent oestrogenic effects in the body, and these are likely to be a contributing factor in the development of breast cancer. ${ }^{2}$ Dicophane (DDT), which is stored in the body's fatty tissue as dichlorodiphenyldichloroethylene (DDE), and polychlorinated phenols are two such pesticides.
Fuchs has reviewed some of the evidence linking pesticides and breast cancer. ${ }^{3}$ Dr Mary Wolff, of the Mount Sinai School of Medicine, measured blood concentrations of DDE in women and found that women with high concentrations were four times as likely to have breast cancer as those with lower concentrations. Another study, published in the Archives of Environmental Health in 1993, found that patients with breast cancer had more than twice the concentration of polychlorinated phenols and DDE in their breast tissue as comparable women without cancer. Mortality from breast cancer declined in Israel after dicophane and other carcinogenic pesticides, which occurred in high concentrations in milk and dairy products, were banned in 1978 .

There is strong evidence that "oestrogen dominance" is a major risk factor for breast cancer and that progesterone has a protective effect. ${ }^{4}$ In a prospective study by Cowan et al premenopausal women with low progesterone concentrations were found to have 5.4 times the risk of developing premenopausal breast cancer of those with normal concentrations and to have a 10 -fold increase in deaths from all malignant neoplasms. There is a good case for measuring at least serum progesterone concentrations in women at high risk and in those with breast cancer and considering progesterone supplementation if concentrations are low. Synthetic progestogens have very different effects on the body from progesterone itself. Natural progesterone (derived from the Mexican yam and available commercially) can be conveniently given as a transdermal cream or sublingual drops and is the compound of choice in restoring hormone concentrations in such women.

PETER J LEWIS Complementary physician

Centre for the Study of Complementary Medicine,

Southampton,

Hampshire SO15 2DT

1 McPherson K, Steel CM, Dixon JM. Breast cancer-epidemiology, risk factors, and genetics. $B M \mathcal{F}^{1}$ 1994;309:1003-6. (15 October.)

2 Raloff J. Ecocancers: do environmental factors underlie a breast cancer epidemic? Science News 1993;144:10-3.

3 Fuchs $\mathrm{K}$. The pesticide and breast cancer connection. Women's Health Letter $1994 \mathrm{Jan}$

4 Lee JR. Natural progesterone. Sebastopol, CA: BLL Publishing, 1993.

5 Cowan LD, Gordis L, Tonascia JA, Jones GS. Breast cancer incidence in women with progesterone deficiency. $A m$ Epidemiol 1981;114:209-17.

\section{Mammography after treatment for breast cancer}

EDITOR,-Eva Grunfeld and colleagues believe that they may have identified a widespread problem relating to the follow up of patients with breast cancer. ${ }^{1}$ In fact, all they have identified is the inadequacy of a survey by non-clinicians analysing a single factor identified in a patient's notes without taking heed of clinical features.

In most large breast units mammography is included in the follow up protocol for patients who have received treatment for breast cancer. On many occasions, however, mammography that may have been planned sometime in advance becomes inappropriate owing to a change in clinical circumstances, including the development of cytologically or histologically proved recurrent disease, distant metastases, or unrelated coexisting medical problems. On occasions mammography is delayed because of continued induration of the breast after surgery and radiotherapy. In addition, patients may be disinclined to undergo further mammography. Accordingly, Grunfeld and colleagues' concern that "over a third of patients did not undergo mammography at the expected time" is unfounded without further analysis of the reasons for the delay. A clinical protocol does not fail because clinical circumstances may preclude adherence to it.

Breast Clinic,

Northampton General Hospital NHS Trust,

Northampton NN1 5BD

1 Grunfeld E, Mant D, Vessey MP, Cole DJ, Stewart JA. Mammography after treatment for breast cancer. $B M \mathcal{F} 1994$; 309:665-6. (10 September.)

\section{Author's reply}

EDITOR,-S J A Powis is concerned that we did not consider clinical circumstances in our definition of delay. This is not correct. All the medical records in the 145 cases were reviewed by a clinician, and the review took into account the complete clinical picture according to the medical records. When a delay in mammography was found no clinical circumstances that could explain why it had occurred was evident on this review. None of the patients had recurrent disease or distant metastases, which Powis cites as possible reasons for not performing mammography. In addition, almost all of these patients had had mammography at least once after completing primary treatment, making it unlikely that mammography was subsequently postponed until the resolution of induration from surgery or radiotherapy.

It is possible that in a small proportion of patients the delay was due to a coexisting medical problem or the patient's choice. To allow for these factors we reported delays of six and 12 months. This allowed clinicians reading the report to decide which interval they thought was clinically important.

We clearly stated the limitations of our findings -that they were restricted to two hospitals and 145 patients-and that we cannot say for certain that we have identified a more widespread problem. We also clearly stated that the benefit and optimum frequency of mammographic surveillance remain uncertain and should be the subject of a randomised trial (we recognise the methodological difficulties of such a trial). We are concerned, however, that it may be neither a clinical decision nor the patient's choice that decides whether a policy of mammographic surveillance adopted by a clinician gets implemented. Rather, it may be the unfortunate consequence of breast cancer clinics being overburdened.

EVA GRUNFELD

Department of Medicine, University of Ottawa, Assistant profess Ottawa, Ontario, Canada

\section{Medical staffing in general hospitals}

EDITOR,-I support E R Williams and colleagues' proposal for a core unit in hospitals 1 -a model similar to the facilitating unit ${ }^{2}$ - but believe that this alone will not be sufficient in the future. Other innovations need consideration in addition.

Firstly, the respective roles of general practitioners and hospital specialists must be clarified. The NHS is unique in having a primary care infrastructure, but we do not seem to utilise its potential fully. Thus, given that general practitioners are the guardians of patients' health and have the relevant histories, why do junior doctors spend time clerking patients and why cannot such histories be shared by means of the existing information technology?

Secondly, we need to review the place of traditional hospital specialties. Why do we continue to have separate medical and surgical gastroenterology services or rheumatology and orthopaedic services, for example?

Overall we need to decide what care should be 
provided in primary care and what in secondary care and to ensure services are oriented to specific health problems through appropriate resource allocation, protocols, and team working. This is urgent since health commissioners are already shifting resources into primary care while hospitals are facing increasing activity.

Thirdly, not all hospital care has to be delivered by highly trained consultants, and alternatives such as doctors of subconsultant grade, midwives, nurse practitioners, and physiotherapists need exploration. Because patients with low back pain were faced with a one year wait for an outpatient consultation a clinic led by a nurse was established in Middlesbrough. Although formal evaluation is awaited, the reports from general practitioners have been complementary and waiting times have fallen to 8-10 weeks; in this regard, common waiting lists, which can ensure equity, are worth considering. ${ }^{3}$

Finally, we need to consider the concept of the 24 hour hospital not only for reasons of medical staffing but also because of advances whereby medical technologies and facilities become rapidly outdated before we get value for money.

A major review of the way in which we deliver health care and train doctors is required. We should start by developing a full agenda and thinking laterally and not get constrained by traditional models.

RAJAN MADHOK South of Tyne Health Commission, South Shields,

Tyne and Wear NE33 3BN

1 Williams R, Harvey LP, Ingrams G, Robin PE, Temple JG. Back to basics: medical staffing in general hospitals. $B M F$ 1994;309. 1166-7. (29 October.)

2 Madhok R, Holtby I. Facilitating unit: better management of acute admissions. Health Care Management 1989;4:19-21.

3 Madhok R. Cutting waiting lists. BMF 1991;302:532.

\section{Purchasing vascular services}

EDITOR,-The data in Malcolm Forsyth's letter commenting on the development of specialised vascular units add little to the argument because they make no concession to two important factors related to survival after surgery for aortic aneurysm-namely, mode of presentation and selection of patients. ${ }^{1}$ A large proportion of patients who present as an emergency decreases overall survival while a small proportion improves it. A better test of a unit's ability to provide safe care lies in the outcome after elective repair of aortic aneurysm.

These points are illustrated by a comparison of Forsyth's data collected over six years with our experience at Guy's and Lewisham Hospitals over the past three years. We performed 158 operations; $20(13 \%)$ patients died and $138(87 \%)$ survived. This uncritical use of statistics makes our results seem encouraging. Proper analysis shows a large number of elective operations (73 with three deaths) compared with non-elective operations ( 85 with 17 deaths). Superficially, our non-elective surgery has a $20 \%$ mortality. The non-elective surgery is performed in two distinct subgroups of cases- "urgent" without frank rupture (51 with four deaths) and emergencies with frank rupture (34 with 13 deaths). These groups are statistically distinct $(\mathbf{P}<0.01)$.

Even detailed reports rarely include the number of patients who reach the casualty department but are refused emergency surgery. Selection of the most hardy patients by long ambulance trips to specialist units can also bias reported results.

Finally, we have doubts about the accuracy of codes based on the International Classification of Diseases. We maintain a computer and handwritten database of all our vascular procedures. Comparison of these data with coding according to the classification shows agreement in only $70 \%$ of cases. Thirty two patients were incorrectly coded as having repairs of an aneurysm, and $30 \%$ of aneurysms were not coded at all.

These points are important. The inappropriate publication of clinical results has potential political dangers. There is a risk that misleading figures might be used in the formulation of inappropriate health care policy or that politicians will recognise the superficiality of such analysis, disregard all of the data, and ignore potentially important health care issues.

M R TYRRELL Senior registrar Y P PANAYIOTOPOULOS Lecturer PRTAYLOR

Department of Surgery, ascular surgeon Guy's Hospital, London SE1 9RT

1 Forsythe M. Purchasing vascular services. BMF 1994;309:881. (1 October.)

\section{The inverse pay law}

EDITOR,-I have just discovered a new medical law. The new law is called "the inverse pay law" and stands alongside and may partly explain the well recognised "inverse care law." The new law states that the more needy a patient is and the more difficult it is to look after him or her the less one gets paid for doing so.

My practice looks after a large number of opiate misusers and people who misuse alcohol and other substances. We look after the bail hostel and homeless people in Bedford. We look after six hostels; these are refuges and rehabilitation facilities. We also look after many people with marginal lifestyles in the community. For looking after one hostel we get paid the equivalent of a clinical assistant's fee for one session, and for looking after another hostel we get paid the equivalent of half a session. For many hundreds of patients in the community and the care of the four other hostels we get paid capitation fees. Many of these patients cause a huge workload: the average consultation rate of an opiate misuser is 36 consultations a year. We have calculated that, taking into account our set running costs, we pay for looking after many of these patients rather than being paid.

The only problem about my new law is that it could disappear if ever a government that recognised that these people really needed care and was prepared to pay for it was elected. Nevertheless, I consider this eventuality remote in the extreme.

E E J MARTIN

Bedford MK40 3NG General practitioner

\section{Rural general practitioners and out of hours cover}

\section{The need for a humane contract}

Edrror,-As the General Medical Services Committee seems to have ceased to represent the interests of rural general practitioners, I would like to open a debate on out of hours cover. Recent reports on the death of a 26 year old junior doctor who died after an 86 hour week said that he was "about to enter the calmer waters of general practice." As a general practitioner who has spent most of the past 20 years in a rural practice of three doctors, with no possibility of sharing out of hours work, I have worked a (roughly) 40 hour week and shared cover on the remainder, making 80 hours in all. Included has been one weekend in three lasting 77 hours. Whenever on call, night or day, we must be ready to go immediately and fully equipped to patients who have had a heart attack and other emergencies.

Four fifths of general practitioners have expressed an interest in opting out of their 24 hour commitment, ${ }^{1}$ but the GMSC has failed to implement this mandate, which will need to be done vigorously because the government refuses even to negotiate it. After all, it's cheap: the government's recent pricing of about $£ 3000$ per doctor would pay me $£ 3$ an hour for night cover and visits. ${ }^{2}$

The GMSC's policy of retaining 24 hour commitment on a fairly uniform remuneration basis works only for those who can abrogate 24 hour continuity by using locum services or large cooperatives. Meanwhile we in more rural areas are leftto die quietly? A humane contract is not impossible: the Danes have one.

MARTIN LAWRENCE General practitioner

Chipping Norton OX7 5AA

1 Electoral Reform Ballot Services. Your choices for the future: survey of GP opinion. UK report. London: Electoral Reform Ballot Services, 1992.

2 Beecham L. Out of hours deal rejected by general practitioners. $B M \mathcal{F}$ 1994;309:1392. (26 November.)

\section{New scheme is ill conceived}

EDITOR,-I read with dismay the new proposals for payments for night visits by general practitioners. ${ }^{\prime}$ The new scheme is ill conceived and dogmatically flawed and does not answer the concerns of the profession with regard to the stresses of continued 24 hour responsibility. If the government insists on forcing through and imposing these proposals it should not be assisted by our negotiators under the "Munich mentality." If the proposals are unacceptable to most general practitioners-and I believe that that is the casewe should say so and refuse to cooperate with their implementation.

I am a single handed practitioner with a list of 2400; an average of 150 night visits arise each year. I find it hard to believe that many general practitioners have only 51 night visits a year and that these general practitioners will be the sole winners in the new scheme. I have calculated that the income for night visits over the past few years has decreased for exactly the same amount of work (from $£ 9300$ before 1988 to $£ 3300$ under the new scheme). This cannot be justified. This entails being on call for over 110 hours a week. The rate for additional night cover under the present arrangements is a paltry $£ 1.76$ an hour and under the new scheme would be $86 \mathrm{p}$ an hour.

I agree with those colleagues who wish to drop their 24 hour responsibility; there should be a modest reduction in income for those who wish to follow that route, which should reflect the appalling standards of pay for this onerous duty, which contributes so much to the stresses of general practice. For those general practitioners who wish or have to retain 24 hour responsibility, however, remuneration should adequately reflect the hours and work entailed.

This scheme acts as an incentive not to do night calls and to hand the responsibility over to deputising services but insists on general practitioners retaining 24 hour responsibility for their patients while the remuneration is lowered. In effect this means that we are all subsidising the provision of 24 hour care and that the highest contribution is made by those general practitioners who continue to do their own calls. This is bad for the profession.

H C ROBINSON

Penclawdd Health Centre, Principal in general practic

Swansea SA4 3YN

1 Beecham L. Out of hours deal rejected by general practitioners. $B M F$ 1994;309:1392. (26 November.) 